\title{
Health Assistant Bot
}

\author{
Tarun Rao ${ }^{1 *}$, Bharath Kumar Dasa ${ }^{2}$, Dhruva Gupta ${ }^{1}$, Monisha Bhattacharya ${ }^{3}$, Sheena Siddiqui ${ }^{4}$ \\ ${ }^{I}$ Accenture PLC, Bangalore, India \\ ${ }^{2}$ Accenture PLC, Chennai, India \\ ${ }^{3}$ Accenture PLC, Kolkatta, India \\ ${ }^{4}$ Accenture PLC, Delhi, India
}

*Corresponding Author: Tarun Rao, Accenture PLC, Bangalore, India

\begin{abstract}
In the era of growing mobile devices importance of a chatbot is paramount due to its ease of use. And due to the Covid 19 pandemic gross insufficiency of resources has also come to light. In such testing times an application which can help with diagnosis of any disease online through a chatbot is of great help. In this paper deep learning is used to predict whether or not a person is prone to a heart attack based on certain features. And this model is deployed and is made accessible to via a chatbot.
\end{abstract}

Keywords: Chatbot, Deep Learning.

\section{INTRODUCTION}

Standing in long queues in hospitals and waiting to see a doctor has long been a problem facing people suffering from any ailment. It has been observed that even after a doctor has prescribed a test at a diagnostic center, one has to repeat this process of waiting in long queues to show the medical report to any doctor to seek their opinion. The solution to this problem can be found in Artificial Intelligence.

Artificial Intelligence can be used to predict whether or not a person suffers from a certain disease based on symptoms. In addition to this one can develop a chatbot which can facilitate in patients avoiding standing in long queues in meeting doctors. Chatbots can be used to diagnose whether a person with certain symptoms require more medical care or not. Chatbots can make use of Artificial Intelligence to suggest basic medicines for generic ailments. This would also lead to lessening the burden on healthcare systems across the globe.

Cardiovascular is a major illness that needs special attention. In today's fast lifestyle one can suffer cardiovascular disease due to factors ranging from age, smoking habits, blood pressure fluctuations, glucose etc.

In this paper the medical chatbot aligns with serving two main objectives. The first objective is to build a machine learning model which shall help in predicting whether or not a person is prone to heart attack in the near future. This model shall be integrated with a chatbot to enable the interaction with people having a doubt on their medical status.

The novelty of this paper lies in the fact that Artificial Neural networks shall be used to predict whether or not a person is prone to suffer from a heart attack in the next 10 years. The said model shall be integrated with a chatbot that shall facilitate people directly conversing with a chatbot and to find out whether they are prone to a heart attack in the near future.

Paper organization: The rest of the paper is organized as follows: Section 2 presents the main components of a chatbot framework. Section 3 deals with explain regarding machine learning and artificial neural networks. Section 4 deals with results. Section 5 concludes the findings followed by future work and references to publications cited in this paper

\section{2. СНатвот}

A chatbot is a software application used to conduct an on-line chat conversation via text or text-tospeech, in lieu of providing direct contact with a live human agent. Chatbots can be built using many proprietary frameworks like Google dialog flow, Microsoft BOT framework, Amazon lex etc..[1][2]

In this paper Google dialoglflow framework shall be used to build bots. The architecture used in this paper is [3]: 


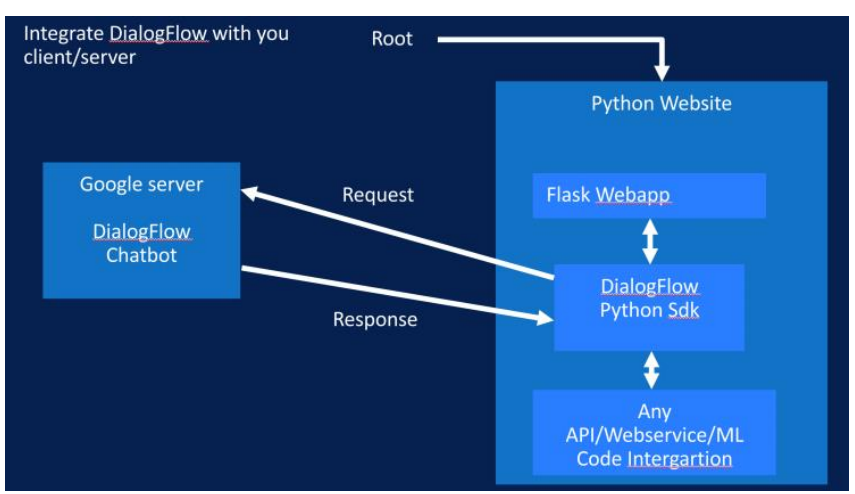

Fig. Chatbot Architecture

As depicted in the above architecture the end users conversation is directed using a web browser in the form of http request. The request reaches the Goggle dialogflow agent which in turn responds back with a response as stored in the Google server[4][5]. The following component's of Google dialogflow are involved in creating the chatbot:

\subsection{Agent}

Agents are created on the dialogflow platform which store all the conversations flowing between the end user and the response to the said conversations based on the context. This agent can be accessed from the web application wherein the chatbot is deployed using an api access token.

\subsection{Intent}

Intent is the objective that the end user wants to fulfill via the conversation with chatbot agent

\subsection{Entity}

Entities modify intents. Entities are used for the agent to understand the end user conversation and respond based on a particular key word. These key words are defined using entities.[6][7][8]

\section{NeURAL NeTWORKS}

Machine learning is the study of computer algorithms that improve automatically through experience. It can be categorized into following categories[9]:

- Supervised Learning: These algorithms are used when data is labelled.

- Unsupervised Learning: These algorithms are used when data is unlabeled.

- Reinforcement Learning: These algorithms are used when very less data is available. In this case the reinforcement learning agent learn from environment.

There are many algorithms designed under each of these categories. Artificial Neural Networks is one such algorithm which can be used inn machine learning. Neural network is a network of artificial neuron connected in a layered fashion as shown below[10][11]:

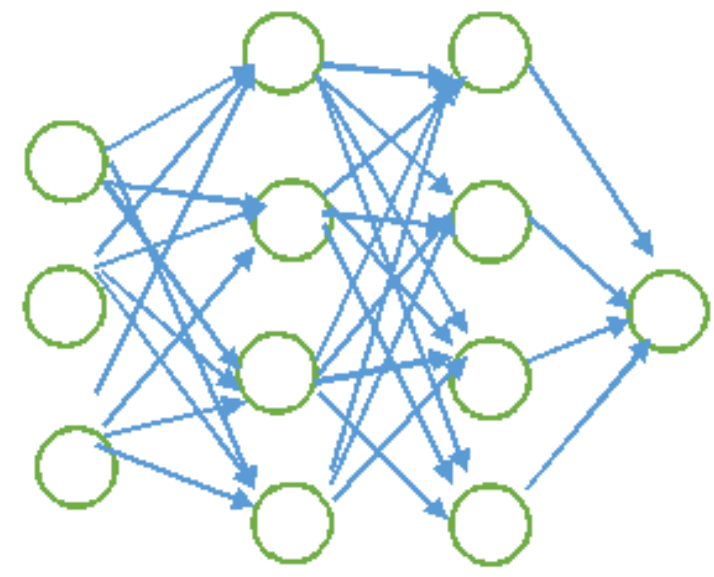

Fig1. Neural Network 
Neural networks have a layered architecture with every layer being made of artificial neurons. It has one input layer, one output layer and one or more hidden layers. Every input to a neuron in NN has a weight. Weight increases the steepness off activation function whereas bias is used to delay triggering of activation function. Activation function of a neuron defines the output of a neuron given its inputs. Few activation functions used in neural networks are given below[12][13]14]:

\subsection{Linear Activation Function:}

It takes the form:

$\mathrm{A}=\mathrm{CX}$

However, the main disadvantage with this activation function is that it cannot be used for backpropagation.

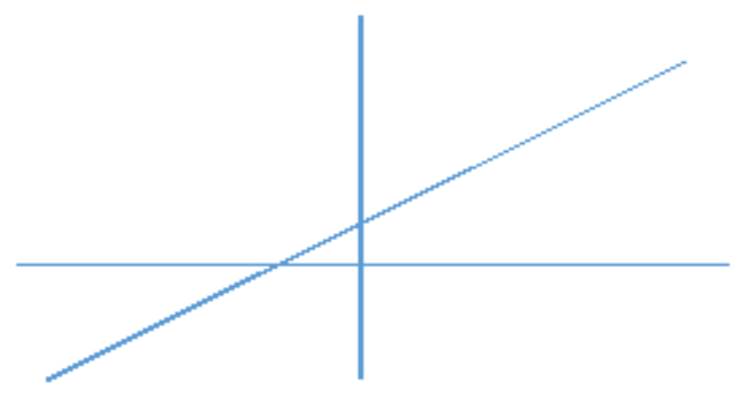

Fig2. Linear Activation function

\subsection{Sigmoid/Logistic Activation function:}

It has output values between 0 and 1 . However it suffers from the problem of vanishing gradient.

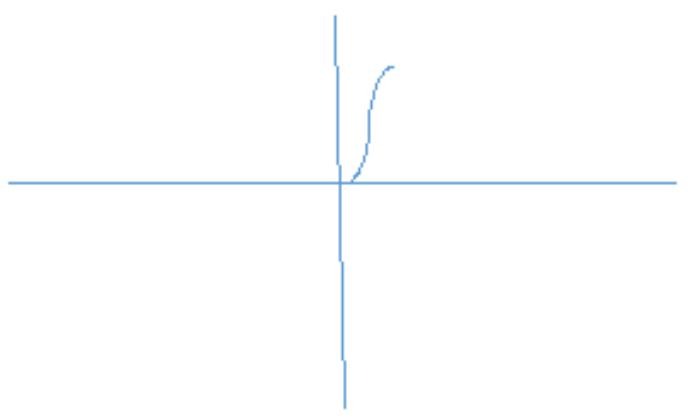

Fig3. Sigmoid activation function

\subsection{Tanh / Hyperbolic Tangent:}

This is zero centered and it makes it easier to model inputs which are positive negative or neutral values.

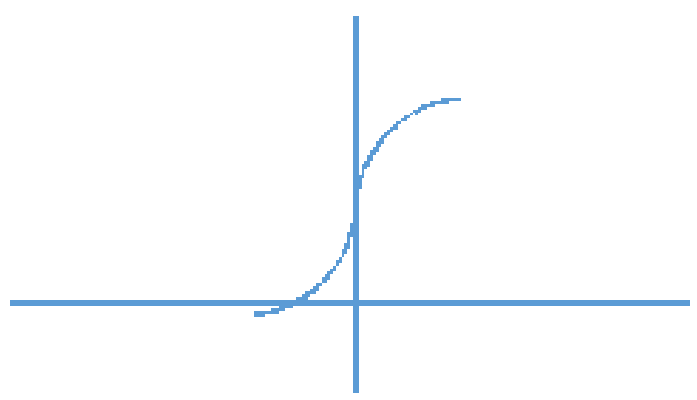

Fig4. Tanh Activation function

\subsection{Rectified Linear Unit:}

It allows the neural network to converge quickly. However when inputs approach negative or zero the gradient becomes zero due to which it cannot perform backpropogation. 


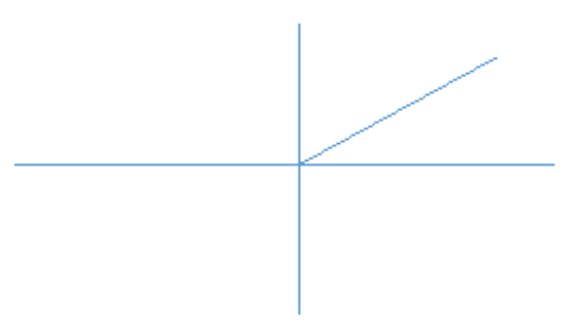

Fig5. Relu Activation Function

\subsection{Leaky Relu:}

Thia activation function has a small slope in the negative direction and hence allows bacxkpropogation

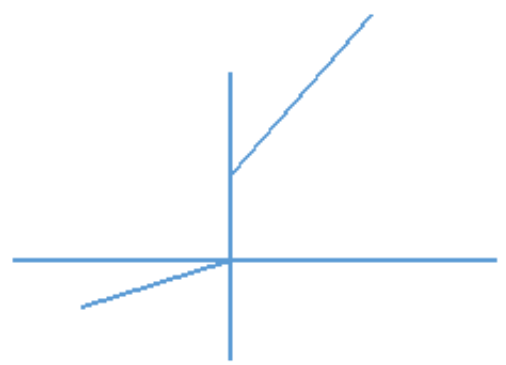

Fig6. Leaky Relu Activation Function

There are different architectures of neural network which are in use today. Few of the prominent neural network architectures are discussed below[15][16]:

\subsection{Convolution Neural Network:}

It has the below mentioned architecture:

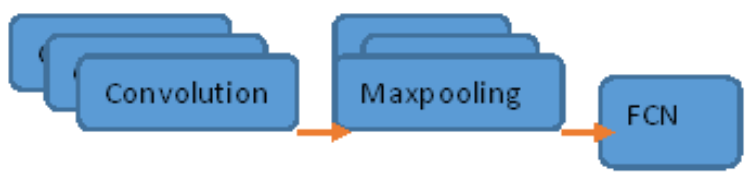

In the convolution layer the input is convolved and the input is passed on to the next layer. In the pooling layer the dimensions of the input are reduced by combining the output of clusters into a single neuron. In the fully connected network one neuron in one layer is connected to every neuron in the subsequent layer. Its functioning is similar to any other neural network.

\subsection{Recurrent Neural Network:}

It has two major properties:

- It has distributed hidden state that allows them to store a lot of information about the past

- Non-Linear dynamics that allow them to update their hidden state in complicated ways.

There are different types of RNN architectures. Few of them along with their applications are mentioned below:
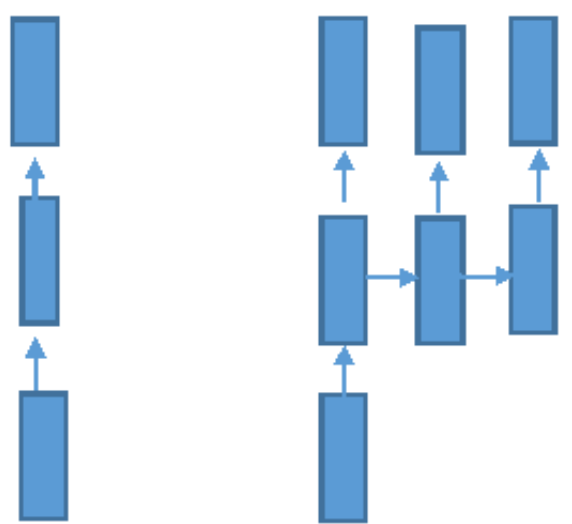

Fig 7a) one to one RNN b) One to many $R N N$ 


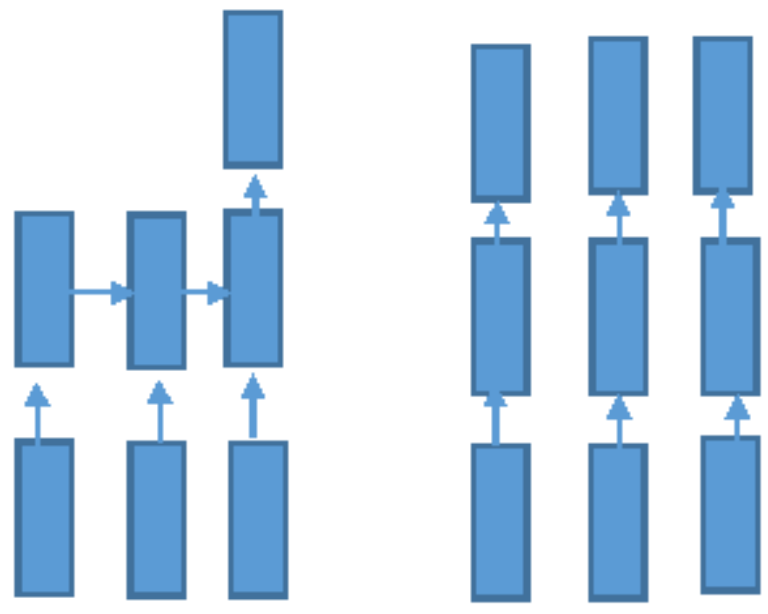

Fig 8a) Many to one RNN b) Many to Many RNN

Applications of the above architectures are:

- One to One RNN is used in image classification

- One to many RNN is used in image captioning

- Many to one RNN is used in sentiment analysis

- Many to many RNN is used in video classification.

In this paper a fully connected neural network was used to predict whether a person is prone to a heart ailment or not.

\section{ReSUlts}

The dataset consists of 16 features which are used for predicting heart diseases[17][19].

- Gender - It accepts 2 values 0 for Female and 1 for Male.

- Age - It accepts an integer of value ranging from 0-100

- Education of the user -

- $1=$ Some High School

- 2 = High School or GED

- $3=$ Some College or Vocational School

- $4=$ College

- CurrentSmoker flag: It accepts 2 values 0 for No-Smoker and 1 for Smoker.

- Cigarettes per day : Accepts the count of number of cigarettes per day

- BP meds : it accepts two values 0 for not on BP medications, 1 for on BP medications.

- Prevalent stroke : It accepts to value 0 for no prevalent stroke and 1 for prevalent stroke

- Prevalent Hypertension : It accepts to value 0 for no prevalent hypertension and 1 for prevalent hypertension

- Diabetes : It accepts to value 0 for not diabetic and 1 for diabetic.

- Total Cholesterol: Accepts an integer value of the amount of Cholesterol in the body. (mg/dL)

- Systolic blood pressure : Accepts an integer value from the range of 83 to $235 \mathrm{mmHg}$

- Diastolic blood pressure : Accepts an integer value from the range of 45 to $145 \mathrm{mmHg}$

- BMI : Body Mass Index calculated as- Weight (kg)/ Height(meter-squared)

- Heart Rate - Beats/Min (Ventricular)

- Glucose - Accepts an integer value from the range from 40 to $395 \mathrm{mg} / \mathrm{dL}$ 
The objective of this paper is to predict whether or not a person is prone to heart attack based on above mentioned features. Neural networks have been used to build this model. And this model has been deployed using a python flask application and has also been integrated with Google Dialogflow chatbot[18]. PFB few screenshots of the same:

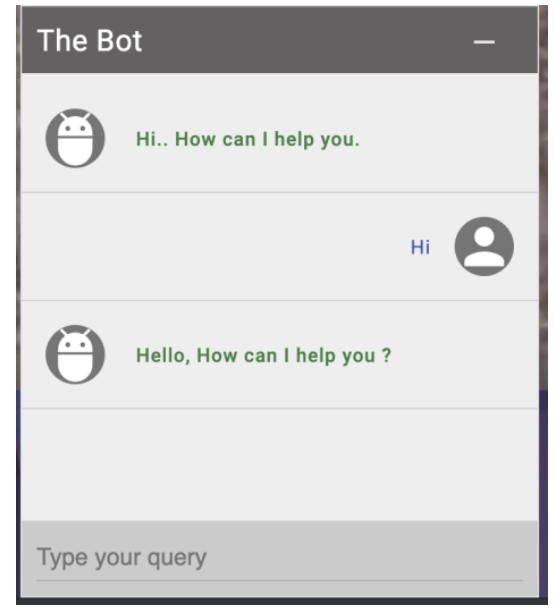

Then the user interacts with the bot stating that he/she is keen to understand about the heart health condition. The bot on return replies it is happy to assist and would require few details.

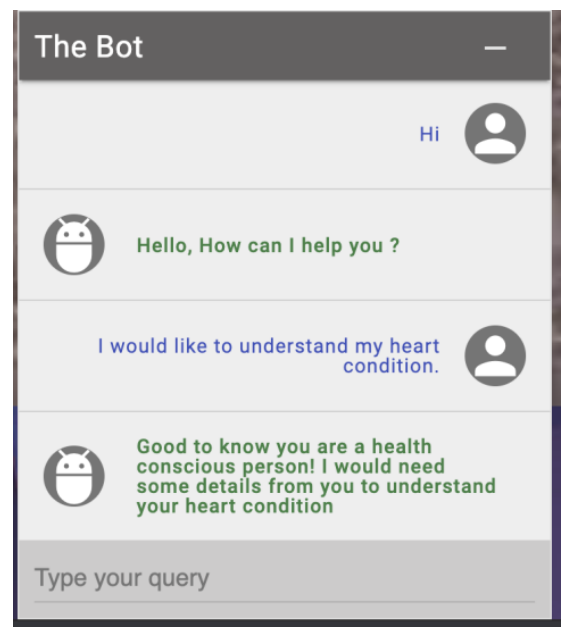

The user then agrees to provide the details that are required to assess the heart health condition. The bot checks for the gender and age. The user enters the details.

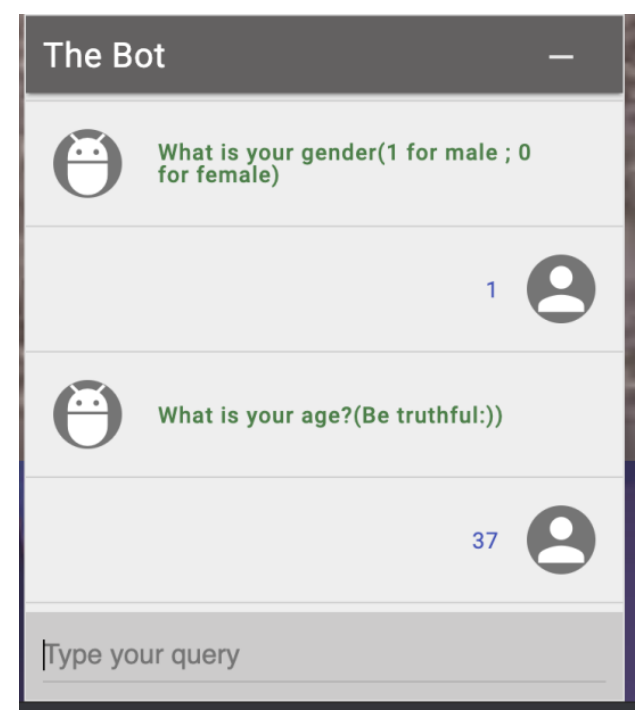

The bot checks for the education qualification and whether the user is a current smoker. Similarly, other feature information about the user is collected and run through the neural network model. The final. Screenshot is: 


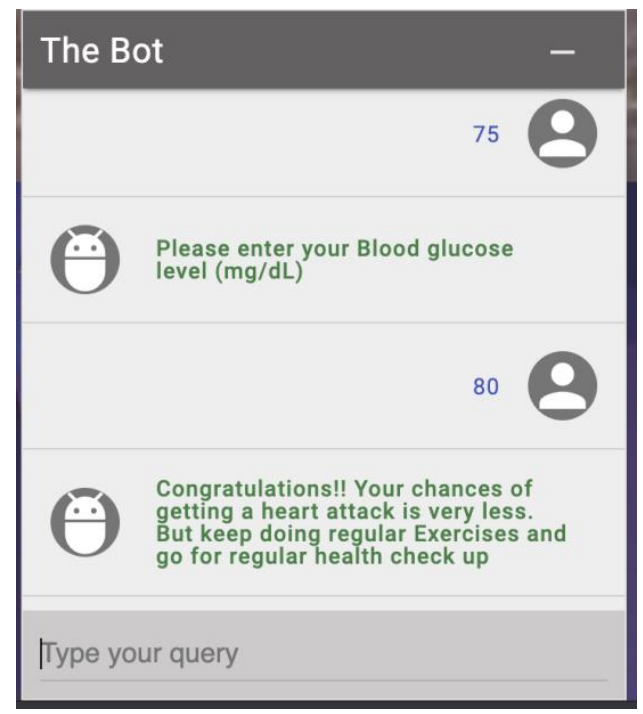

Once collecting all the information, it sends all the values for the respective features to the already trained Machine learning model and based on the output of the model it displays the message if the user would be prone to cardiac risk or not. In this case the user is not having any risk based on the given inputs as per the prediction.

\section{CONClusion}

Neural Networks can be used to build a model which can diagnose any particular disease. It can be integrated with any application using a chatbot framework. In times of a pandemic like Covid 19 such applications can be of great help in times when Government does not have necessary resources in doing excessive testing.

\section{REFERENCES}

[1] Eleonora Pantano, Gabriele Pizzi, Forecasting artificial intelligence on online customer assistance: Evidence from chatbot patents analysis, Journal of Retailing and Consumer Services, Volume 55, 2020, 102096, ISSN 0969-6989,

[2] Alaa A. Abd-alrazaq, Mohannad Alajlani, Ali Abdallah Alalwan, Bridgette M. Bewick, Peter Gardner, Mowafa Househ,An overview of the features of chatbots in mental health: A scoping review, International Journal of Medical Informatics, Volume 132, 2019, 103978, ISSN 1386-5056,

[3] Vijayaraghavan V., Jack Brian Cooper, Rian Leevinson J., Algorithm Inspection for Chatbot Performance Evaluation, Procedia Computer Science, Volume 171, 2020, Pages 2267-2274, ISSN 1877-0509,

[4] Dharun Lingam Kasilingam, Understanding the attitude and intention to use smartphone chatbots for shopping, Technology in Society, Volume 62, 2020 101280, ISSN 0160-791X,

[5] Aleksandra Przegalinska, Leon Ciechanowski, Anna Stroz, Peter Gloor, Grzegorz Mazurek, In bot we trust: A new methodology of chatbot performance measures, Business Horizons, Volume 62, Issue 6, 2019, Pages 785-797, ISSN 0007-6813,

[6] Pavel Smutny, Petra Schreiberova, Chatbots for learning: A review of educational chatbots for the Facebook Messenger, Computers \& Education, Volume 151, 2020, 103862, ISSN 0360-1315,

[7] Mohammad Nuruzzaman, Omar Khadeer Hussain, IntelliBot: A Dialogue-based chatbot for the insurance industry, Knowledge-Based Systems, Volume 196, 2020, 105810, ISSN 0950-7051,

[8] Ben Sheehan, Hyun Seung Jin, Udo Gottlieb, Customer service chatbots: Anthropomorphism and adoption, Journal of Business Research, Volume 115, 2020, Pages 14-24, ISSN 0148-2963

[9] Qiyue Wang, Wenhua Jiao, Peng Wang, YuMing Zhang, A tutorial on deep learning-based data analytics in manufacturing through a welding case study, Journal of Manufacturing Processes, 2020, , ISSN 1526-6125,

[10]Ning Wang, Yuanyuan Wang, Meng Joo Er, Review on deep learning techniques for marine object recognition: Architectures and algorithms, Control Engineering Practice, 2020, 104458, ISSN 0967-0661,

[11] Olga Fink, Qin Wang, Markus Svensén, Pierre Dersin, Wan-Jui Lee, Melanie Ducoffe, Potential, challenges and future directions for deep learning in prognostics and health management applications, Engineering Applications of Artificial Intelligence, Volume 92, 2020, 103678, ISSN 0952-1976,

[12]Fatma Murat, Ozal Yildirim, Muhammed Talo, Ulas Baran Baloglu, Yakup Demir, U. Rajendra Acharya, Application of deep learning techniques for heartbeats detection using ECG signals-analysis and review, Computers in Biology and Medicine, Volume 120, 2020, 103726, ISSN 0010-4825, 
[13] Pranav Puri, Nneka Comfere, Lisa A. Drage, Huma Shamim, Spencer A. Bezalel, Mark R. Pittelkow, Mark D.P. Davis, Michael Wang, Aaron R. Mangold, Megha M. Tollefson, Julia S. Lehman, Alexander Meves, James A. Yiannias, Clark C. Otley, Rickey E. Carter, Olayemi Sokumbi, Matthew R. Hall, Alina G. Bridges, Dennis H. Murphree, Deep Learning for Dermatologists: Part II. Current Applications, Journal of the American Academy of Dermatology, 2020, ISSN 0190-9622,

[14] Shenda Hong, Yuxi Zhou, Junyuan Shang, Cao Xiao, Jimeng Sun, Opportunities and challenges of deep learning methods for electrocardiogram data: A systematic review, Computers in Biology and Medicine, Volume 122, 2020, 103801, ISSN 0010-4825,

[15] Mengshu Ge, Fei Su, Zhicheng Zhao, Dong Su, Deep Learning Analysis on Microscopic Imaging in Materials Science, Materials Today Nano, 2020, 100087, ISSN 2588-8420,

[16] Dennis H. Murphree, Pranav Puri, Huma Shamim, Spencer A. Bezalel, Lisa A. Drage, Michael Wang, Mark R. Pittelkow, Rickey E. Carter, Mark D.P. Davis, Alina G. Bridges, Aaron R. Mangold, James A. Yiannias, Megha M. Tollefson, Julia S. Lehman, Alexander Meves, Clark C. Otley, Olayemi Sokumbi, Matthew R. Hall, Nneka Comfere, Deep Learning for Dermatologists: Part I Fundamental Concepts Journal of the American Academy of Dermatology,2020,ISSN 0190-9622,

[17] Jooyoung Oh, Sooah Jang, Hyunji Kim, Jae-Jin Kim, Efficacy of mobile app-based interactive cognitive behavioral therapy using a chatbot for panic disorder, International Journal of Medical Informatics, Volume 140, 2020,104171, ISSN 1386-5056

[18] https://cloud.google.com/dialogflow/docs

[19] https://www.kaggle.com/amanajmera1/framingham-heart-study-dataset

Citation: Tarun Rao et.al., (2020). "Health Assistant Bot”, International Journal of Research Studies in Computer Science and Engineering (IJRSCSE), 7(2), pp.1-8. DOI:http://dx.doi.org/10.20431/23494859.0702001

Copyright: () 2020 Authors, This is an open-access article distributed under the terms of the Creative Commons Attribution License, which permits unrestricted use, distribution, and reproduction in any medium, provided the original author and source are credited. 